\title{
On the numerical range of some block matrices with scalar diagonal blocks. ${ }^{\text {th }}$
}

\author{
Titas Geryba and Ilya M. Spitkovsky \\ Division of Science and Mathematics, New York University Abu Dhabi (NYUAD), \\ Saadiyat Island, P.O. Box 129188 Abu Dhabi, United Arab Emirates
}

\begin{abstract}
Several new verifiable conditions are established for matrices of the form $\left[\begin{array}{cc}\alpha I_{n-k} & C \\ D & \beta I_{k}\end{array}\right]$ to have the numerical range equal the convex hull of at most $k$ ellipses. For $k=2$, these conditions are also necessary, provided that the ellipses are co-centered.
\end{abstract}

Keywords: Numerical range

\section{Introduction}

As usual, let $\mathbb{C}$ denote the field of complex numbers, $\mathbb{C}^{n \times m}$ stand for the set of all $n$-by- $m$ matrices with complex entries, with this notation abbreviated to just $\mathbb{C}^{n}$ when $m=1$. The standard scalar product on $\mathbb{C}^{n}$ will be denoted $\langle, .$,$\rangle , with the respective norm \|$.$\| defined by \|x\|=\langle x, x\rangle^{1 / 2}$.

The numerical range (a.k.a. the field of values or the Hausdorff set) of a matrix $A \in \mathbb{C}^{n \times n}$ is defined as

$$
W(A)=\left\{\langle A x, x\rangle: x \in \mathbb{C}^{n},\|x\|=1\right\} .
$$

Introduced a century ago in the pioneering work by O. Toeplitz [13] and Hausdorff [7], it has been researched extensively thereafter, see e.g. [6] or [9,

The results are partially based on the Capstone project of the first named author [TG] under the supervision of the second named author [IMS]. This work was supported in part by Faculty Research funding from the Division of Science and Mathematics, New York University Abu Dhabi.

Email addresses: tg1404@nyu.edu [TG]; imspitkovsky@gmail.com, ims2@nyu.edu, ilya@math.wm.edu [IMS] 
Chapter 1]. Being the image of the unit sphere of $\mathbb{C}^{n}$ under a continuous mapping $f_{A}: x \mapsto\langle A x, x\rangle$, the numerical range $W(A)$ is a closed, bounded, and connected subset of $\mathbb{C}$. Moreover, $W(A)$ is convex (the classical ToeplitzHausdorff theorem). More specifically, it is the convex hull of the so called numerical range generating curve $C(A)$ having the following characteristic property: for every angle $\theta$ there are exactly $n$ tangent lines of $C(A)$, counting multiplicities, forming the angle $\theta$ with the positive direction of $x$-axis, and their intercepts with the orthogonal family of lines are exactly the eigenvalues of $\operatorname{Im}\left(e^{-i \theta} A\right)$. A more detailed description of the generating curve can be found in [10], where the notion was introduced, or its English translation [11]; see also [5].

Here and below for any square matrix $X$

$$
\operatorname{Re} X=\frac{1}{2}\left(X+X^{*}\right) \text { and } \operatorname{Im} X=\frac{1}{2 i}\left(X-X^{*}\right)
$$

are the hermitian components from the representation

$$
X=\operatorname{Re} X+i \operatorname{Im} X
$$

In this paper we will consider exclusively matrices of the form

$$
A=\left[\begin{array}{cc}
\alpha I_{n-k} & C \\
D & \beta I_{k}
\end{array}\right]
$$

with their diagonal blocks being scalar multiples of the identity. Of course, for $n=2$ all matrices have form (1), while $W(A)$ in this case is an elliptical disk with the foci at the eigenvalues of $A$, degenerating - for normal $A$-into the line segment connecting the eigenvalues (the Elliptical Range theorem). Our goal is to see the extent to which this shape of $W(A)$ persists for matrices (1) in higher dimensions.

More specifically, we are interested in the cases when the numerical range of $A$ is the convex hull of a finite number of ellipses.

A known general (but not very constructive) condition for this to occur is stated in Section 2, along with other preliminary results. Several easily verifiable sufficient conditions, applicable to matrices (1), are in Section 3. Finally, if one of the diagonal blocks in (1) is of the size 2-by-2, the criterion is obtained in Section 4 for $W(A)$ to be the convex hull of at most two co-centered ellipses. 


\section{Preliminaries}

Due to an elementary property

$$
W(\omega A+t I)=\omega W(A)+t \text { for any } t, \omega \in \mathbb{C},
$$

it suffices to consider matrices (1) with $\alpha+\beta=0$. We will refrain from doing so in the statements, but will be taking advantage of this simplification in proofs, whenever convenient. The quantity

$$
\gamma=\frac{\alpha-\beta}{2}
$$

invariant under shifts of $A$, will play an important role in what follows.

We will suppose, whenever convenient, that $k \leq n / 2$. This can be done without loss of generality, since an appropriate permutational similarity can be used to switch from (1) to the matrix

$$
\left[\begin{array}{cc}
\beta I_{k} & D \\
C & \alpha I_{n-k}
\end{array}\right]
$$

leaving the numerical range unchanged.

Due to the construction of $C(A)$, the crucial role in the description of $W(A)$ for any matrix $A$ is played by the eigenvalues of $\operatorname{Im}\left(e^{-i \theta} A\right)$. The lemma below provides the pertinent information for matrices of the form (1).

Lemma 1. Let $A$ be of the form (1), with $k \leq n / 2$. Then the eigenvalues of $\operatorname{Im}\left(e^{-i \theta} A\right)$ are $\operatorname{Im}\left(e^{-i \theta} \alpha\right)$, of multiplicity $n-2 k(\geq 0)$, with the remaining $2 k$ given by the formula

$$
\lambda_{j}=\frac{1}{2}\left(\operatorname{Im}\left(e^{-i \theta}(\alpha+\beta)\right) \pm \sqrt{\left(\operatorname{Im}\left(e^{-i \theta}(\alpha-\beta)\right)^{2}+\mu_{j}(\theta)\right.}\right), \quad j=1, \ldots, k
$$

Here $\mu_{j}(\theta)$ are the eigenvalues of the matrix

$$
M(\theta):=H-2 \operatorname{Re}\left(e^{-2 i \theta} Z\right)
$$

with $H$ and $Z$ defined by

$$
H=C^{*} C+D D^{*}, Z=D C .
$$


Proof. As was discussed, it suffices to consider the case $\beta=-\alpha$, in which formula (4) simplifies to

$$
\lambda_{j}= \pm \sqrt{\left(\operatorname{Im}\left(e^{-i \theta} \alpha\right)\right)^{2}+\mu_{j}(\theta) / 4}, \quad j=1, \ldots, k .
$$

The result then follows from the Schur complement formula for the determinant, according to which the characteristic polynomial of $\operatorname{Im}\left(e^{-i \theta} A\right)$ is

$$
\begin{aligned}
& \operatorname{det}\left(\left(\operatorname{Im}\left(e^{-i \theta} \alpha\right)-\lambda\right) I_{n-k}\right) \\
& \cdot \operatorname{det}\left(\left(-\operatorname{Im}\left(e^{-i \theta} \alpha\right)-\lambda\right) I_{k}-\left(\operatorname{Im}\left(e^{-i \theta} \alpha\right)-\lambda\right)^{-1} M(\theta) / 4\right) \\
& \quad=\left(\operatorname{Im}\left(e^{-i \theta} \alpha\right)-\lambda\right)^{n-2 k} \operatorname{det}\left(\left(\lambda^{2}-\left(\operatorname{Im}\left(e^{-i \theta} \alpha\right)\right)^{2}\right) I_{k}-M(\theta) / 4\right) .
\end{aligned}
$$

The eigenvalues $\operatorname{Im}\left(e^{-i \theta} \alpha\right)$ of $\operatorname{Im}\left(e^{-i \theta} A\right)$, if actually present (which is the case when $k<n / 2$ ), correspond to the tangent lines of the numerical range generating curve $C(A)$ all passing through $\alpha$. This means simply that in this case the singleton $\{\alpha\}$ is a component of $C(A)$. For $k>n / 2$ we just need to replace $\alpha$ with $\beta$ in this reasoning.

The remaining tangent lines form a family which is central symmetric with respect to $(\alpha+\beta) / 2$, as (4) implies. Observe also that $\alpha, \beta \in W(A)$, independent of whether or not $\alpha \in C(A)$. We thus arrive at

Proposition 1. Let $A$ be of the form (1). Then $C(A) \backslash\{\alpha, \beta\}$, and thus the numerical range $W(A)$ itself, is central symmetric with respect to $(\alpha+\beta) / 2$.

Note that for $\alpha=\beta(=0)$ this property follows directly from the equality $-A=J A J$, where $J=\operatorname{diag}\left[I_{n-k},-I_{k}\right]$, as observed in [15, Proposition 3.2].

So, if $W(A)$ is the convex hull of finitely many ellipses, some of them may be centered at $(\alpha+\beta) / 2$, with the other coming in pairs, central symmetric with respect to $(\alpha+\beta) / 2$, but centered elsewhere.

The following example shows that such pairs can indeed be present.

Example 1. Let in (1) $\alpha=\beta=0, C=B^{*}+I$ and $D=-I+B$, where $B$ is a 2-by-2 matrix of the form

$$
B=\left[\begin{array}{cc}
a & \sqrt{\left(1-|a|^{2}\right)\left(1-|c|^{2}\right)} \\
0 & c
\end{array}\right]
$$


$|a|=|c| \leq 1$ and $\operatorname{Im} a=\operatorname{Im} c$. Then, as shown in [15, Theorem 3.14], the numerical range of $A$ is a convex hull of two ellipses that are centered away from the origin.
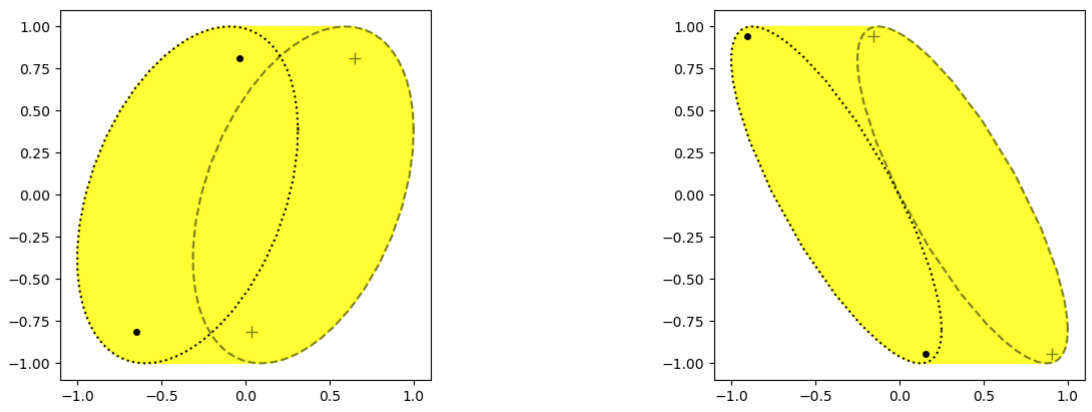

Figure 1: $W(A)$ for example 1. On the left $a=c=\frac{1}{2}+\frac{i}{4}$. On the right $a=c=-\frac{i}{2}$.

In this paper, however, we will restrict our attention to the case when such pairs do not materialize, that is, all the ellipses forming $C(A)$ are co-centered. The following technical lemma will therefore be instrumental.

Lemma 2. Given a matrix $A \in \mathbb{C}^{n \times n}$, its numerical range $W(A)$ is the convex hull of $m$ ellipses centered at the origin if and only if for some partition $\left\{\gamma_{j}\right\}_{j=1}^{m}$ of the unit circle $\mathbb{T}$, the maximal eigenvalue $\lambda_{\max }(\theta)$ of $\operatorname{Im}\left(e^{-i \theta} A\right)$ satisfies

$$
\lambda_{\max }^{2}(\theta)=A_{j} \cos (2 \theta)+B_{j} \sin (2 \theta)+C_{j}, \quad e^{i \theta} \in \gamma_{j},
$$

for some distinct triples $\left\{A_{j}, B_{j}, C_{j}\right\}$ of positive numbers such that $C_{j}>$ $\sqrt{A_{j}^{2}+B_{j}^{2}}, j=1, \ldots, m$.

Before passing to the proof of Lemma 2, let us clarify that for $m=1$ the only element of the partition is the whole circle $\mathbb{T}$, while for $m>1$ each $\gamma_{j}$ is a pair of central symmetric circular arcs, due to the central symmetry of all the ellipses involved.

Proof. From the description of $C(A)$ it easily follows (and also is well known) that the supporting line of $W(A)$ in the direction of $e^{i \theta}$ is passing through the point $e^{i(\theta+\pi / 2)} \lambda_{\max }\left(\operatorname{Im}\left(e^{-i \theta} A\right)\right)$. A direct computation (performed, e.g., 
in the proof of [3, Theorem 1], where the case $m=1$ was treated) shows that the boundary of $W(A)$ contains an arc $E$ of the ellipse with the halfaxes $\sqrt{C \pm \sqrt{A^{2}+B^{2}}}$ and the major axis forming angle $-\frac{1}{2} \tan ^{-1} \frac{B}{A}$ with the positive direction of the $x$-axis if and only if

$$
\lambda_{\max }^{2}(\theta)=A \cos (2 \theta)+B \sin (2 \theta)+C
$$

for all the angles $\theta$ formed by the tangent lines of the elliptical arc $E$ with the positive direction of the $x$-axis. Condition (8) therefore holds if and only if the boundary of $W(A)$ consists of elliptical arcs of exactly $m$ ellipses, perhaps connected by line segments.

\section{Main results}

We will now consider several cases in which the eigenvalues of the matrices $M(\theta)$ given by (5) can be computed explicitly, thus yielding a full description of $C(A)$ and, ultimately, of $W(A)$. All the results will be stated in terms of matrices $H, Z$ defined by (6).

One such case materializes if the matrix $Z$ is normal and commutes with $H$. These matrices can be then diagonalized by the same (unitary) similarity. Let us label the eigenvalues $h_{j}, z_{j}$ of $H$ and $Z$, according to the order in which they appear on the respective diagonals.

Theorem 2. Let the off-diagonal blocks of (1) be such that in (6) the matrix $Z$ is normal and commutes with $H$. Then $W(A)$ is the convex hull of at most $k$ ellipses.

Moreover, each ellipse associated with a particular $\mu_{j}(\theta)$ is centered at $(\alpha+\beta) / 2$ with a major axis parallel to $e^{-i \phi_{j}}$ of length $2 a_{j}$ and minor axis of $2 b_{j}$ which are given by

$$
a_{j}=\left(\frac{|\gamma|^{2}}{2}+\frac{h_{j}}{4}+\frac{1}{2}\left|\gamma^{2}+z_{j}\right|\right)^{\frac{1}{2}}, \quad b_{j}=\left(\frac{|\gamma|^{2}}{2}+\frac{h_{j}}{4}-\frac{1}{2}\left|\gamma^{2}+z_{j}\right|\right)^{\frac{1}{2}} .
$$

Here $\phi_{j}$ is the principal argument of $i \sqrt{\gamma^{2}+z_{j}}$.

Proof. Under the conditions imposed on $H$ and $Z$, the matrix (5) is diagonalizable for all values of $\theta$ by the same similarity as $Z$ and $H$, implying that

$$
\mu_{j}(\theta)=h_{j}-2 \operatorname{Re}\left(e^{-2 i \theta} z_{j}\right)=h_{j}-2 \operatorname{Re}\left(z_{j}\right) \cos 2 \theta-2 \operatorname{Im}\left(z_{j}\right) \sin 2 \theta .
$$


According to Lemma 1 the equation of $\lambda_{j}(\theta)$ corresponding to $A-((\alpha+\beta) / 2) I$ is given by:

$$
\begin{aligned}
\lambda_{j}^{2} & =\operatorname{Im}\left(e^{-i \theta} \gamma\right)^{2}+\mu_{j}(\theta) / 4 \\
& =\operatorname{Im}(\gamma)^{2} \cos ^{2} \theta+\operatorname{Re}(\gamma)^{2} \sin ^{2} \theta-\operatorname{Re}(\gamma) \operatorname{Im}(\gamma) \sin 2 \theta+\mu_{j}(\theta) / 4 \\
& =\frac{|\gamma|^{2}}{2}+\frac{h_{j}}{4}-\frac{\operatorname{Re}\left(z_{j}+\gamma^{2}\right)}{2} \cos 2 \theta-\frac{\operatorname{Im}\left(z_{j}+\gamma^{2}\right)}{2} \sin 2 \theta
\end{aligned}
$$

By Lemma 2 the above corresponds to an ellipse centered at the origin with minor and major axes as described in the statement. Translating these ellipses by $(\alpha+\beta) / 2$ we obtain the result.

Remark 1. Proof of Theorem 2 goes through if we merely suppose that the matrices $Z, Z^{*}$ and $H$ can be put in a triangular form by a simultaneous similarity. However, this (formally weaker) requirement in fact implies that $Z$ is normal and commutes with $H$.

Conditions of Theorem 2 hold in particular when $Z$ is a scalar multiple of the identity matrix. Then all $z_{j}$ are the same, implying that the ellipses described by Theorem 2 form a nested family. Therefore, independent of the value of $k, W(A)$ is an elliptical disk. More specifically:

Corollary 1. Let the off-diagonal blocks of (1) be such that $D C=c I$. Then $W(A)$ is an elliptical disk centered at $(\alpha+\beta) / 2$ with the axes of length $\sqrt{\|H\|+2|\gamma|^{2} \pm 2\left|\gamma^{2}+c\right|}$, and the major axis parallel to $e^{-i \phi}$, where $\phi$ is the (principal) argument of $i \sqrt{\gamma^{2}+c}$.

The description of $W(A)$ simplifies even further when $c=0$, i.e., $D C=0$. The axes of the ellipse $W(A)$ then have length $\sqrt{\|H\|^{2}+4|\gamma|^{2}}$ and $\|H\|$.

Condition $D C=0$ in geometrical terms may be recast as the kernel of $D$ containing the range of $C$. This happens, in particular, when the $j$-th row of $C$ consists of all zeros provided that the $j$-th column of $D$ is non-zero. This covers Scenario 2 from [3, Theorem 7], corresponding to $j=1$.

Of course, $D C=0$ when $D=0$. The latter case is listed as Scenario 1 in [3, Theorem 7]. Note however that all quadratic matrices (i.e., matrices with minimal polynomial of degree 2) can be reduced to this case via a unitary similarity - the approach used in [14] when proving the ellipticity of the numerical ranges of such matrices. 
Another setting obviously guaranteeing $D C=c I$ is when $k=1$. The ellipticity of $W(A)$ in this case was proved in [12, see also [3, Theorem 2].

Yet another situation in which conditions of Theorem 2 hold is when there exist unitary $U \in \mathbb{C}^{k \times k}$ and $V \in \mathbb{C}^{(n-k) \times(n-k)}$ such that $U D V$ and $U C^{*} V$ are both diagonal. Indeed, then matrices (6) are simultaneously diagonalizable under the unitary similarity $X \mapsto U X U^{*}$. This case, along with a number of situations reducing to it, was extensively treated in [1]. It was also observed in [1] that the existence of such $U, V$ is equivalent to normality of two matrices, $C D$ and $D C$, see, e.g., [8, p. 426]. The normality of $D C$ of course holds when $k=1$ while there is no reason for $C D$ to also be normal.

Situations with $Z=D C$ being normal and commuting with $H$, while $C D$ is not normal, do materialize in more interesting settings as well. Consider for example the case $n=4, k=2$,

$$
D=\left[\begin{array}{cc}
\xi & \eta \\
\bar{\eta} & \zeta
\end{array}\right] U \text { and } C=D^{-1}\left[\begin{array}{cc}
\omega & 0 \\
0 & \bar{\omega}^{-1}
\end{array}\right]
$$

with $U \in \mathbb{C}^{2 \times 2}$ unitary, $\xi, \zeta>0, \xi \zeta-|\eta|^{2}=1, \xi \neq \zeta$, and $|\omega| \neq 1$.

Then

$$
Z=D C=\left[\begin{array}{cc}
\omega & 0 \\
0 & \bar{\omega}^{-1}
\end{array}\right]
$$

is normal, while a direct computation shows that $H=Z^{*}\left(D D^{*}\right)^{-1} Z+D D^{*}$ is diagonal, and therefore commutes with $Z$. At the same time $C D=D^{-1} Z D$ is unitarily similar to

$$
\left[\begin{array}{cc}
\zeta & -\eta \\
-\bar{\eta} & \xi
\end{array}\right] Z\left[\begin{array}{cc}
\xi & \eta \\
\bar{\eta} & \zeta
\end{array}\right]=\left[\begin{array}{cc}
* & \eta \zeta\left(\omega-\bar{\omega}^{-1}\right) \\
-\bar{\eta} \xi\left(\omega-\bar{\omega}^{-1}\right) & *
\end{array}\right] .
$$

The off-diagonal entries of the resulting matrix differ in magnitude, precluding it from being normal. Consequently, the matrix $C D$ is not normal either.

Here is a particular numerical example, to illustrate.

Example 2. Consider $C$ and $D$ as follows:

$$
C=\frac{1}{2}\left[\begin{array}{cc}
8 & -1 \\
-4 & 1
\end{array}\right] \quad D=\left[\begin{array}{ll}
1 & 1 \\
1 & 2
\end{array}\right]
$$

Then

$$
D C=\frac{1}{2}\left[\begin{array}{ll}
4 & 0 \\
0 & 1
\end{array}\right] \text { is normal, while for } C D=\frac{1}{2}\left[\begin{array}{cc}
7 & 6 \\
-3 & -2
\end{array}\right]
$$


we have

$$
(C D)^{*} C D-C D(C D)^{*}=\frac{1}{4}\left[\begin{array}{cc}
-27 & 81 \\
81 & 27
\end{array}\right] \neq 0 .
$$

Finally, for this choice of $C, D$ :

$$
H=\frac{1}{4}\left[\begin{array}{cc}
8 & -4 \\
-1 & 1
\end{array}\right]\left[\begin{array}{cc}
8 & -1 \\
-4 & 1
\end{array}\right]+\left[\begin{array}{ll}
1 & 1 \\
1 & 2
\end{array}\right]^{2}=\left[\begin{array}{cc}
22 & 0 \\
0 & 5.5
\end{array}\right] .
$$

is indeed diagonal.
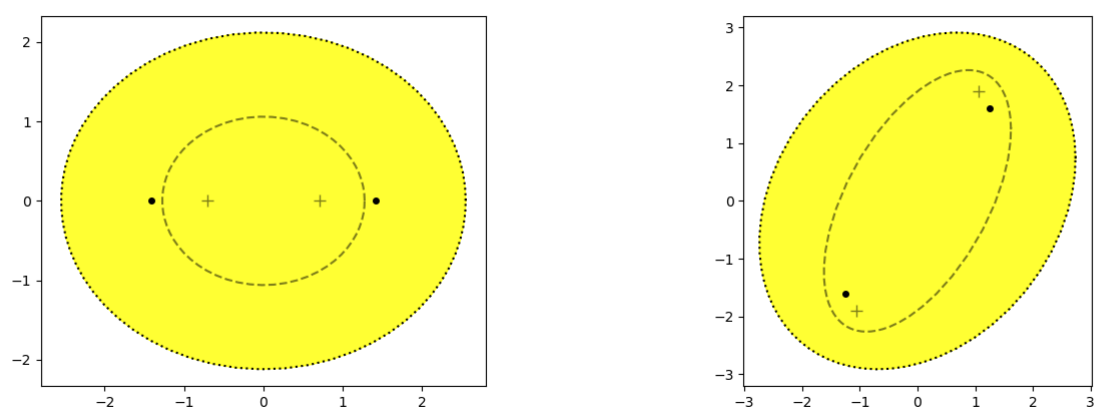

Figure 2: $W(A)$ from Example $2 \alpha=\beta=0$ on the left and $\alpha=-\beta=1+2 i$ on the right.

As can be seen from Corollary 1 , in the setting of Theorem 2 the number $m(A)$ of ellipses actually involved in generating $W(A)$ may be strictly smaller than its upper bound $k$ (unless, of course, $k=1$ ). A moment's thought reveals that $m(A)$ is the number of distinct functions (9) attaining the maximal (with respect to $j$ ) value while $\theta$ varies in $[0, \pi]$.

Determining the value of $m(A)$ in the setting of Theorem 2 without imposing additional restrictions on the matrices (6) may be rather challenging. Here is yet another case in which it can be done. Recall that a matrix $X$ is essentially Hermitian if it is normal with a spectrum lying on a line (equivalently: if $W(X)$ has empty interior).

Corollary 2. Let $Z$ be essentially Hermitian. Suppose in addition that $H$ is a scalar multiple of the identity, while $Z$ is not. Then $W(A)$ is the convex hull of two non-nested ellipses, both centered at $(\alpha+\beta) / 2$ with their major (and thus minor) axes forming the right angle. 
Proof. Under the conditions imposed on $H$ and $Z$, from (9) it follows that

$$
\mu_{s}(\theta)-\mu_{t}(\theta)=2 \operatorname{Re}\left(e^{-2 i \theta}\left(z_{t}-z_{s}\right)\right)
$$

with the arguments of all the non-zero differences $z_{t}-z_{s}$ being equal $\bmod \pi$. So, the switch in the sign of $\mu_{s}(\theta)-\mu_{t}(\theta)$ for all $s, t=1, \ldots, k$ such that $z_{t} \neq z_{s}$ does actually occur. Moreover, it happens at the same two values of $\theta$, different by $\pi / 2$.

In particular, the above corollary holds when $Z$ is Hermitian or SkewHermitian. The following is such an example. Consider:

$$
A=\left[\begin{array}{cc}
0 & -I_{k}+B \\
I_{k}+B^{*} & 0
\end{array}\right]
$$

where $B$ is unitary. In this case, we obtain that $H=4 I_{k}$ and $Z=B^{*}-B$. Since $H$ is a scalar multiple of the identity, and $Z$ is skew-Hermitian, we conclude that $W(A)$ is the convex hull of two ellipses.

The exactly same reasoning applies to the case of $A$ given by

$$
A=\left[\begin{array}{cc}
0 & -I_{k}^{\prime}+B \\
I_{k}^{\prime}+B^{*} & 0
\end{array}\right]
$$

where $I_{k}^{\prime}$ is the identity $k$-by- $k$ matrix with the last column deleted, and $B \in \mathbb{C}^{k, k-1}$ is an isometry. This case was considered in [15, Theorem 3.5], see also [2, Theorem 3.4].

We now turn to the situation when the eigenvalues $\mu_{j}(\theta)$ do not depend on $\theta$ in spite of the fact that the matrices $M(\theta)$ themselves do.

Theorem 3. In the notation (6), suppose that, for some subspace $L$ invariant under $H$, the range of $Z$ is contained in $L$ which in turn is contained in the kernel of $Z$. Then $W(A)$ is an elliptical disk centered at $(\alpha+\beta) / 2$ with major axis parallel to $e^{-i \phi}$ of length $\sqrt{\mu+4|\gamma|^{2}}$ and minor axis of length $\sqrt{\mu}$, where $\mu$ is the largest eigenvalue of $H-2 \operatorname{Re} Z, \gamma=(\alpha-\beta) / 2$ and $\phi$ is the (principal) argument of $i \gamma$.

Proof. With respect to the decomposition $\mathbb{C}^{n}=L \oplus L^{\perp}, H$ and $Z$ can be expressed as:

$$
H=\left[\begin{array}{cc}
H_{1} & 0 \\
0 & H_{2}
\end{array}\right], \quad Z=\left[\begin{array}{cc}
0 & Z_{0} \\
0 & 0
\end{array}\right],
$$


and therefore

$$
M(\theta)=H-2 \operatorname{Re}\left(e^{-2 i \theta} Z\right)=\left[\begin{array}{cc}
H_{1} & -e^{-2 i \theta} Z_{0} \\
-e^{2 i \theta} Z_{0}^{*} & H_{2}
\end{array}\right]=U^{*} M U,
$$

where $U$ is the unitary matrix $\operatorname{diag}\left[I_{n-k},-e^{2 i \theta} I_{k}\right]$, and the matrix

$$
M=\left[\begin{array}{ll}
H_{1} & Z_{0} \\
Z_{0}^{*} & H_{2}
\end{array}\right]
$$

is independent of $\theta$. Consequently, the eigenvalues $\mu_{j}$ of $M(\theta)$ are the same as those of $M$, and thus also do not depend on $\theta$.

By Lemma 1 applied to $A_{0}=A-\frac{\alpha+\beta}{2} I$, the respective eigenvalues $\lambda_{j}(\theta)$ satisfy

$$
\begin{aligned}
\lambda_{j}^{2} & =\left(\operatorname{Im}\left(e^{-i \theta} \gamma\right)\right)^{2}+\mu_{j} / 4 \\
& =(\operatorname{Im} \gamma)^{2} \cos ^{2} \theta+(\operatorname{Re} \gamma)^{2} \sin ^{2} \theta-\operatorname{Re} \gamma \operatorname{Im} \gamma \sin 2 \theta+\mu_{j} / 4 \\
& =\frac{\mu_{j}}{4}+\frac{|\gamma|^{2}}{2}-\frac{\operatorname{Re}\left(\gamma^{2}\right)}{2} \cos 2 \theta-\frac{\operatorname{Im}\left(\gamma^{2}\right)}{2} \sin 2 \theta .
\end{aligned}
$$

Consequently, in the notation of Lemma 2 ,

$$
\lambda_{\max }^{2}(\theta)=\frac{\mu}{4}+\frac{|\gamma|^{2}}{2}-\frac{\operatorname{Re}\left(\gamma^{2}\right)}{2} \cos 2 \theta-\frac{\operatorname{Im}\left(\gamma^{2}\right)}{2} \sin 2 \theta,
$$

implying (by the same Lemma) that $W\left(A_{0}\right)$ is the elliptical disk centered at the origin with major axis parallel to $e^{-i \phi}$ of length $\sqrt{\mu+4|\gamma|^{2}}$ and minor axis of length $\sqrt{\mu}$. Translating the matrix $A_{0}$ by $(\alpha+\beta) / 2$ we obtain the result.

Conditions imposed on $Z$ by Theorem 3 imply that it is nilpotent, namely $Z^{2}=0$. If $H$ is a scalar multiple of the identity, then this is also sufficient. Another sufficient condition is $C D=0$. Indeed, setting $L=\operatorname{ker} C$ and making use of the fact that the range of $D$ lies in $L$, we then obtain the following matrix representations:

$$
C=\left[\begin{array}{ll}
0 & C_{12} \\
0 & C_{22}
\end{array}\right], \quad D=\left[\begin{array}{cc}
D_{11} & D_{12} \\
0 & 0
\end{array}\right]
$$

with respect to the decomposition $L \oplus L^{\perp}$. Direct computations show then

$$
Z=\left[\begin{array}{cc}
0 & D_{11} C_{12}+D_{12} C_{21} \\
0 & 0
\end{array}\right], \quad H=\left[\begin{array}{cc}
D_{11} D_{11}^{*}+D_{12} D_{12}^{*} & 0 \\
0 & C_{12}^{*} C_{12}+C_{22}^{*} C_{22}
\end{array}\right]
$$


implying that indeed so chosen $L$ is invariant under $H$ and contains the range of $Z$.

This case covers the remaining Scenario 3 of [3, Theorem 7] in which $C$ has the first zero column while $D$ is a matrix with all rows being zero except maybe the first one.

Note, however, that the same conclusion can be reached by passing from (1) to $(3)$ and invoking the result for the case $D C=0$ treated earlier.

In case of square matrices $C, D$ (i.e., when $k=n / 2$ ), the nilpotency of $Z$ implies that $C$ and $D$ cannot both be invertible. The following class of examples shows that one of them still can.

Let $k=n / 2$, choose $C$ having non-zero pair-wise orthogonal columns, and let $D=J C^{-1}$, where $J$ has the block structure $\left[\begin{array}{ll}0 & T \\ 0 & 0\end{array}\right]$ with an arbitrary $k$-by- $k$ block $T$. Then of course $Z=J$, the matrix $C^{*} C$ is diagonal and invertible, implying the block diagonal structure of $H=C^{*} C+J\left(C^{*} C\right)^{-1} J^{*}$. So, conditions of Theorem 3 are satisfied by $L$ being the span of the first $k$ vectors $e_{1}, \ldots, e_{k}$ of the standard basis. A numerical example is below.

Example 3. Consider $C$ and $D$ as follows:

$$
C=\left[\begin{array}{cc}
1+i & 1+i \\
1 & -2
\end{array}\right], \quad D=\frac{1}{6}\left[\begin{array}{cc}
1-i & -2 \\
0 & 0
\end{array}\right] \text {. }
$$

We have then:

$$
Z=\left[\begin{array}{ll}
0 & 1 \\
0 & 0
\end{array}\right], \quad H=\frac{1}{6}\left[\begin{array}{cc}
19 & 0 \\
0 & 36
\end{array}\right],
$$

and $L=\operatorname{Span}\left\{e_{1}\right\}$ does the job.
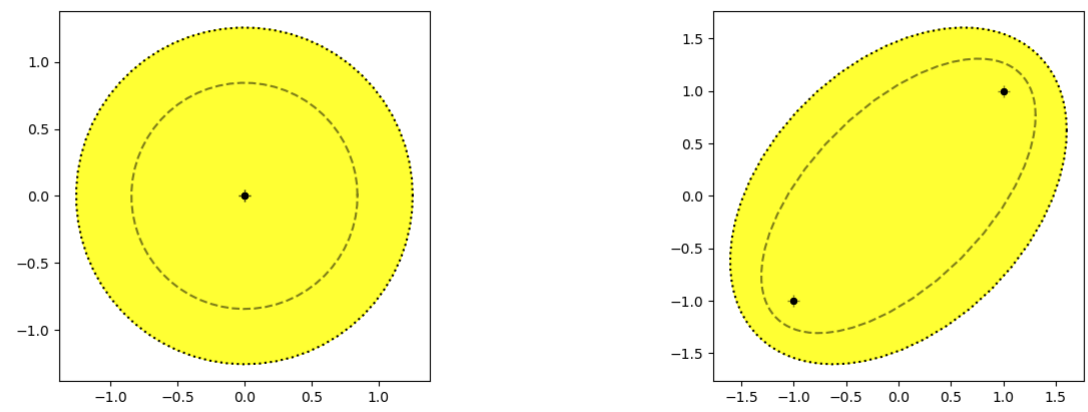

Figure 3: $W(A)$ from example 3, $\alpha=\beta=0$ on the left and $\alpha=-\beta=1+i$ on the right. 


\section{The $k=2$ case}

In this section, we concentrate on the case $k=2$. Independent of the value of $n(\geq 4)$, matrices (6) are then of 2-by-2 size, and the eigenvalues $\mu_{j}(\theta)$ of (5) can be found explicitly by solving a quadratic equation. Namely, denoting by $h_{j}$ and $z_{j}$ the eigenvalues of $H$ and $Z$, respectively, and by $z_{i j}$ the entries of $Z$ in the orthonormal basis of the eigenvectors of $H$ (by convention choosing $Z$ to be diagonal when $H$ is a scalar multiple of the identity):

$$
\mu_{1,2}=\frac{1}{2}\left(h_{1}+h_{2}-2 \operatorname{Re}\left(z_{11}+z_{22}\right) \cos 2 \theta-2 \operatorname{Im}\left(z_{11}+z_{22}\right) \sin 2 \theta \pm \sqrt{\Delta}\right)
$$

where $\Delta$ is given by:

$$
\begin{aligned}
\Delta & =\left(h_{1}-h_{2}\right)^{2}+2\left|z_{22}-z_{11}\right|^{2}+4\left|z_{12}\right|^{2}+4\left|z_{21}\right|^{2} \\
& +4\left(h_{1}-h_{2}\right) \operatorname{Re}\left(z_{22}-z_{11}\right) \cos 2 \theta \\
& +4\left(h_{1}-h_{2}\right) \operatorname{Im}\left(z_{22}-z_{11}\right) \sin 2 \theta \\
& +2 \operatorname{Re}\left(\left(z_{1}-z_{2}\right)^{2}\right) \cos 4 \theta \\
& +2 \operatorname{Im}\left(\left(z_{1}-z_{2}\right)^{2}\right) \sin 4 \theta
\end{aligned}
$$

Theorem 4. Let in (1) $k=2$. Then $W(A)$ is the convex hull of ellipses centered at $(\alpha+\beta) / 2$ if and only if at least one of the following conditions holds:

(i) $Z$ is normal commuting with $H$,

(ii) eigenvalues of $Z$ coincide, and it shares an eigenvector with $H$,

(iii) $H$ and $Z$ are such that

$$
-\left(h_{1}-h_{2}\right)^{2} \frac{z_{12} z_{21}}{\left(z_{1}-z_{2}\right)^{2}}=\left(\left|z_{12}\right|+\left|z_{21}\right|\right)^{2}
$$

Observe that conditions (i) and (ii) hold simultaneously if and only if $Z$ is a scalar multiple of the identity, cases (ii) and (iii) are mutually exclusive, and "(i) but not (ii)" is a subcase of (iii). Examples 3 and 2 show, respectively, that situations "(ii) but not (i)" and "(i) but not (ii)" indeed materialize. As for "(iii) but not (i)", the respective examples also exist. Their construction is more involved, however, and can be achieved by solving certain non-linear matrix equations with the use of [4]. This issue will be addressed elsewhere. 
Proof. According to (10) and (4), $\lambda_{1}^{2}(\theta)+\lambda_{2}^{2}(\theta)$ is a real linear combination of $\cos 2 \theta, \sin 2 \theta$ and 1 . Consequently, each of $\lambda_{j}^{2}$ is such a linear combination if and only if their difference is. Equivalently, this happens if and only if $\Delta$ has the form $(a \cos 2 \theta+b \sin 2 \theta+c)^{2}$ for some $a, b, c \in \mathbb{R}$.

As it can be seen from (11), this in its turn is equivalent to the system

$$
\begin{array}{r}
a+i b= \pm 2\left(z_{1}-z_{2}\right), \\
(a+i b) c=2\left(h_{1}-h_{2}\right)\left(z_{22}-z_{11}\right), \\
c^{2}=\left(h_{1}-h_{2}\right)^{2}+2\left|z_{22}-z_{11}\right|^{2}+4\left|z_{12}\right|^{2}+4\left|z_{21}\right|^{2}-2\left|z_{1}-z_{2}\right|^{2} .
\end{array}
$$

It therefore remains to show that one of the conditions (i)-(iii) holds if and only if the system (13) is consistent. To this end, consider several possibilities separately, keeping in mind that

$$
\left(z_{1}-z_{2}\right)^{2}=\left(z_{11}-z_{22}\right)^{2}+4 z_{12} z_{21}
$$

Case 1. $z_{1}=z_{2}, h_{1}=h_{2}$, implying immediately that (ii) holds. On the other hand, the solution of $(13)$ is delivered by

$$
a=b=0, \quad c= \pm\left(2\left|z_{22}-z_{11}\right|^{2}+4\left|z_{12}\right|^{2}+4\left|z_{21}\right|^{2}\right)^{1 / 2} .
$$

Case 2. $z_{1}=z_{2}, z_{11}=z_{22}$. According to (14), this is only possible when $z_{12} z_{21}=0$, implying that $H$ and $Z$ share a common eigenvector. So, (ii) holds again. And the system $(13)$ is consistent, having

$$
a=b=0, \quad c= \pm\left(\left(h_{1}-h_{2}\right)^{2}+4\left|z_{12}\right|^{2}+4\left|z_{21}\right|^{2}\right)^{1 / 2}
$$

as its solution.

Case 3. $z_{1}=z_{2}$, while $h_{1} \neq h_{2}, z_{11} \neq z_{22}$. Then $Z$ is not normal, because the only normal matrices with coinciding eigenvalues are scalar multiples of the identity. Also, (14) implies that $z_{12} z_{21} \neq 0$, and therefore $H$ and $Z$ do not have eigenvectors in common. Consequently, neither of (i)-(ii) holds. And (iii) does not hold either, since the left hand side of (12) is undefined.

On the other hand, the system (13) is inconsistent. Indeed, the first equation implies that $a=b=0$, which is in contradiction with the second equation.

Case 4. $z_{1} \neq z_{2}$. The solution of $(13)$, if exists, is given by

$$
a= \pm 2 \operatorname{Re}\left(z_{1}-z_{2}\right), b= \pm 2 \operatorname{Im}\left(z_{1}-z_{2}\right), c= \pm \frac{\left(h_{1}-h_{2}\right)\left(z_{22}-z_{11}\right)}{z_{1}-z_{2}}
$$


(with the coordinated choice of the three signs), as follows from the first two equations.

Subcase $4 A$. $h_{1}=h_{2}$ or $z_{11}=z_{22}$. Then $c=0$, and in order for (15) to indeed deliver a solution, according to the third equation it is necessary and sufficient that

$$
\left(h_{1}-h_{2}\right)^{2}+2\left|z_{22}-z_{11}\right|^{2}+4\left|z_{12}\right|^{2}+4\left|z_{21}\right|^{2}-2\left|z_{1}-z_{2}\right|^{2}=0 .
$$

If $z_{11}=z_{22}$, formula (14) allows to substitute $\left|z_{1}-z_{2}\right|^{2}$ by $4\left|z_{12} z_{21}\right|$, and so (16) can be rewritten as

$$
\left(h_{1}-h_{2}\right)^{2}+4\left(\left|z_{12}\right|-\left|z_{21}\right|\right)^{2}=0 .
$$

So, condition $z_{11}=z_{22}$ implies $h_{1}=h_{2}$, and we may concentrate on the latter.

According to 16 then:

$$
2\left|z_{22}-z_{11}\right|^{2}+4\left|z_{12}\right|^{2}+4\left|z_{21}\right|^{2}=2\left|z_{1}-z_{2}\right|^{2}
$$

Comparing with (14) we see that this happens if and only if

$$
\left|z_{12}\right|=\left|z_{21}\right|, \quad 2 \arg \left(z_{11}-z_{22}\right)=\arg z_{12}+\arg z_{21} \quad \bmod 2 \pi
$$

(the condition on the arguments being imposed only when it makes sense, i.e., $z_{11} \neq z_{22}, \quad z_{12} z_{21} \neq 0$ ).

But this is exactly the normality criterion for $Z$. Consequently, (i) holds. Observe that $Z$ is not a scalar multiple of the identity since $z_{1} \neq z_{2}$.

Subcase $4 B$. $h_{1} \neq h_{2}$. Then, as shown above, $z_{11} \neq z_{22}$, and in order for $c$ from (15) to be real, it is necessary and sufficient that

$$
\arg \left(z_{11}-z_{22}\right)=\arg \left(z_{1}-z_{2}\right) \quad \bmod \pi
$$

Also, plugging in this value of $c$ into the third equation of $(13)$ yields

$$
\frac{\left(h_{1}-h_{2}\right)^{2}\left(z_{22}-z_{11}\right)^{2}}{\left(z_{1}-z_{2}\right)^{2}}=\left(h_{1}-h_{2}\right)^{2}+2\left|z_{22}-z_{11}\right|^{2}+4\left|z_{12}\right|^{2}+4\left|z_{21}\right|^{2}-2\left|z_{1}-z_{2}\right|^{2} .
$$

Taking (14) into consideration, this simplifies to 


$$
-2\left(h_{1}-h_{2}\right)^{2} \frac{z_{12} z_{21}}{\left(z_{1}-z_{2}\right)^{2}}=\left|\left(z_{1}-z_{2}\right)^{2}-4 z_{12} z_{21}\right|-\left|z_{1}-z_{2}\right|^{2}+2\left|z_{12}\right|^{2}+2\left|z_{21}\right|^{2}
$$

Since the right hand side of $(18)$ is non-negative, this equality hold only if

$$
\frac{z_{12} z_{21}}{\left(z_{1}-z_{2}\right)^{2}} \leq 0
$$

The latter inequality, when combined with $(14)$, yields

$$
\left|z_{11}-z_{22}\right|^{2}=\left|z_{1}-z_{2}\right|^{2}+4\left|z_{12} z_{21}\right|
$$

which allows to simplify $(18)$ further to $(12)$.

On the other hand, (12) implies (19) (this is clear if $h_{1} \neq h_{2}$, and can be arranged by putting $Z$ in a triangular form if this is not the case). Along with (14), this guarantees (17). Thus, (13) admits a real solution.

We have exhausted all possible cases, thus completing the proof.

Since $k=2$, for matrices $A$ satisfying conditions of Theorem 4 their generating curve $C(A)$ consists of two ellipses. Depending on whether or not these ellipses are nested, the numerical range $W(A)$ itself will either be an elliptical disk, or the convex hull of two non-nested ellipses with the same center, thus having four flat portions on its boundary. The next statement describes when which of the two possibilities occurs.

Theorem 5. Let the matrix A satisfy conditions of Theorem 4. Then $W(A)$ is the convex hull of two non-nested ellipses in case (i), if in addition $\left|h_{1}-h_{2}\right|<$ $2\left|z_{1}-z_{2}\right|$, and just an elliptical disk in all other cases.

Proof. For matrices $A$ satisfying either of the conditions (i)-(iii) of Theorem 4, formulas (10) for the eigenvalues of $M(\theta)$ given by (5) take the form:

$$
\begin{aligned}
\mu_{1,2}=\frac{1}{2}\left(h_{1}+h_{2}-2\right. & \operatorname{Re}\left(z_{11}+z_{22}\right) \cos 2 \theta \\
& \left.-2 \operatorname{Im}\left(z_{11}+z_{22}\right) \sin 2 \theta \pm(a \cos 2 \theta+b \sin 2 \theta+c)\right),
\end{aligned}
$$

where $a, b, c$ solve 13 .

The respective ellipses are nested if and only if the difference $\mu_{1}(\theta)-\mu_{2}(\theta)$ does not change the sign, in other words, if and only if

$$
4\left|z_{1}-z_{2}\right|^{2}=a^{2}+b^{2} \leq c^{2} .
$$


This immediately takes care of case (ii), in which $a=b=0$, and thus (21) holds. Case (i) is also straightforward. Indeed, in this case $c^{2}=\left(h_{1}-h_{2}\right)^{2}$, so that (21) is equivalent to $2\left|z_{1}-z_{2}\right| \leq\left|h_{1}-h_{2}\right|$.

It remains to consider case (iii). Using (20), the third formula from (13) can be rewritten as

$$
c^{2}=\left(h_{1}-h_{2}\right)^{2}+4\left(\left|z_{12}\right|+\left|z_{21}\right|\right)^{2} .
$$

From here and 12):

$$
\begin{aligned}
c^{2}=\left(\left|z_{12}\right|+\left|z_{21}\right|\right)^{2} \frac{\left|z_{1}-z_{2}\right|^{2}+4\left|z_{12} z_{21}\right|}{\left|z_{12} z_{21}\right|} & \\
& \geq\left(\left|z_{12}\right|+\left|z_{21}\right|\right)^{2} \frac{\left|z_{1}-z_{2}\right|^{2}}{\left|z_{12} z_{21}\right|} \geq 4\left|z_{1}-z_{2}\right|^{2},
\end{aligned}
$$

implying that 21) does hold.

Note that normal 2-by-2 matrices are essentially Hermitian, and the respective parts of Theorem 5 agree with Corollary 2. Also, for $Z$ nilpotent part (ii) agrees with Theorem 3 .

\section{References}

[1] E. Brown and I. Spitkovsky, On matrices with elliptical numerical ranges, Linear Multilinear Algebra 52 (2004), 177-193.

[2] C.-C. Chang, H.-L. Gau, Y.-S. Wang, S.-C. Wu, and Y.-T. Yeh, Matrices with defect index one, Operators and Matrices 7 (2013), no. 4, 865-885.

[3] M. T. Chien and K.-C. Hung, Elliptical numerical ranges of bordered matrices, Taiwanese J. Math. 16 (2012), no. 3, 1007-1016.

[4] J. C. Engwerda, A. C. M. Ran, and A. L. Rijkeboer, Necessary and sufficient conditions for the existence of a positive definite solution of the matrix equation $X+A^{*} X^{-1} A=Q$, Linear Algebra Appl. 186 (1993), $255-275$.

[5] M. Fiedler, Geometry of the numerical range of matrices, Linear Algebra Appl. 37 (1981), 81-96. 
[6] K. E. Gustafson and D. K. M. Rao, Numerical range. The field of values of linear operators and matrices, Springer, New York, 1997.

[7] F. Hausdorff, Der Wertvorrat einer Bilinearform, Math. Z. 3 (1919), $314-316$.

[8] R. A. Horn and C. R. Johnson, Matrix analysis, Cambridge University Press, New York, 1985.

[9] _ Topics in matrix analysis, Cambridge University Press, Cambridge, 1994, Corrected reprint of the 1991 original.

[10] R. Kippenhahn, Über den Wertevorrat einer Matrix, Math. Nachr. 6 (1951), 193-228.

[11] - On the numerical range of a matrix, Linear Multilinear Algebra 56 (2008), no. 1-2, 185-225, Translated from the German by Paul F. Zachlin and Michiel E. Hochstenbach.

[12] H. Linden, Containment regions for zeros of polynomials from numerical ranges of companion matrices, Linear Algebra Appl. 350 (2002), 125145.

[13] O. Toeplitz, Das algebraische Analogon zu einem Satze von Fejér, Math. Z. 2 (1918), no. 1-2, 187-197.

[14] S.-H. Tso and P. Y. Wu, Matricial ranges of quadratic operators, Rocky Mountain J. Math. 29 (1999), no. 3, 1139-1152.

[15] Y.-T. Yeh, Numerical range of 2-by-2 block matrices, Master's thesis, National Central University, Taiwan, 2011. 